\title{
Deletion of the rpoZ gene, encoding the $\omega$ subunit of RNA polymerase, results in pleiotropic surface- related phenotypes in Mycobacterium smegmatis
}

\author{
Renjith Mathew, Raju Mukherjee, Radhakrishnan Balachandar \\ and Dipankar Chatterji \\ Molecular Biophysics Unit, Indian Institute of Science, Bangalore 560012, India
}

Correspondence

Dipankar Chatterji

dipankar@mbu.iisc.ernet.in

Received 30 January 2006

Revised 6 March 2006

Accepted 6 March 2006

\begin{abstract}
The $\omega$ subunit, the smallest subunit of bacterial RNA polymerase, is known to be involved in maintaining the conformation of the $\beta^{\prime}$ subunit and aiding its recruitment to the rest of the core enzyme assembly in Escherichia coli. It has recently been shown in Mycobacterium smegmatis, by creating a deletion mutation of the rpoZ gene encoding $\omega$, that the physiological role of the $\omega$ subunit also includes providing physical protection to $\beta^{\prime}$. Interestingly, the mutant had altered colony morphology. This paper demonstrates that the mutant mycobacterium has pleiotropic phenotypes including reduced sliding motility and defective biofilm formation. Analysis of the spatial arrangement of biofilms by electron microscopy suggests that the altered phenotype of the mutant arises from a deficiency in generation of extracellular matrix. Complementation of the mutant strain with a copy of the wild-type rpoZ gene integrated in the bacterial chromosome restored both sliding motility and biofilm formation to the wild-type state, unequivocally proving the role of $\omega$ in the characteristics observed for the mutant bacterium. Analysis of the cell wall composition demonstrated that the mutant bacterium had an identical glycopeptidolipid profile to the wild-type, but failed to synthesize the short-chain mycolic acids characteristic of biofilm growth in M. smegmatis.
\end{abstract}

\section{INTRODUCTION}

Bacterial core RNA polymerase (RNAP) is constituted by two $\alpha$ and one each of $\beta, \beta^{\prime}$ and $\omega$ subunits. $\omega$ is the smallest of the subunits, and the least well studied one by far. Even though this subunit was identified to be an integral part of RNAP quite early in RNAP research (Burgess, 1969), it was only recently that its role in the enzyme was elucidated. It has been demonstrated that $\omega$ has a structural role in the enzyme complex, aiding in the folding of $\beta^{\prime}$ and its recruitment to the rest of the enzyme assembly (Ghosh et al., 2001). The crystal structure of Thermus aquaticus RNAP confirmed this function and provided further structural insights into its function (Minakhin et al., 2001; Zhang et al., 1999). However, evidence regarding a functional role played by $\omega$ has been rare in the literature. The finding that a mutation in the rpoZ gene encoding $\omega$ caused pleiotropic phenotypes in Streptomyces kasugaensis was suggestive of functions which required to be further defined (Kojima et al., 2002). A more direct indication has been the recent confirmation of the requirement of the $\omega$ subunit for the response of RNAP to ppGpp (Vrentas et al., 2005).

\footnotetext{
Abbreviations: GPL, glycopeptidolipid; RNAP, RNA polymerase; SEM,
} scanning electron microscopy.
We recently created a deletion mutation of the rpoZ gene encoding the $\omega$ subunit of RNAP in Mycobacterium smegmatis (Mathew et al., 2005). Our experiments demonstrated the physical protection that $\omega$ provides to the largest subunit of RNAP, $\beta^{\prime}$, in $M$. smegmatis. Interestingly, the mutant bacterium also displayed altered colony morphology. It has been noted previously that bacterial colonies growing on solidified media reflect the biofilm mode of growth of the constituent organisms, and indeed constitute a method to study biofilm formation (Branda et al., 2005). After decades of working with planktonic pure cultures microbiologists are increasingly recognizing that in their natural environs bacteria are most often found as adherent multicellular aggregates called biofilms, usually in association with various surfaces and held together by an extracellular matrix. Examples of other common methods used for studying biofilms include submerged biofilms either in flow cells (Christensen et al., 1999) or in batch cultures, commonly in microtitre dishes (O'Toole \& Kolter, 1998), and the floating pellicles that form at the liquid-air interface of standing cultures (Branda et al., 2001; Friedman \& Kolter, 2004; Ojha et al., 2005). Electron micrographs of structured colonies show that they are composed of cells surrounded by an extracellular matrix (Friedman \& Kolter, 2004; Yildiz \& Schoolnik, 1999). Colonies can vary widely in morphology, and there is a clear correlation between 
highly structured morphologies and the ability of a cell to produce an extracellular matrix. Given this, we found it interesting to investigate the biofilm formation and other surface-related phenotypes of our $r p o Z$ mutant strain.

The primary benefit offered to bacteria by the biofilm mode of growth appears to be protection from various environmental insults (Davey \& O'Toole, 2000). It provides the constituent bacterial cells with increased survival ability under stressful conditions such as low nutrients (Gottenbos et al., 1999; Potera, 1996), and the ability of bacteria in biofilms to resist antibiotics has been identified as an increasing problem (Costerton et al., 1999; Mah \& O'Toole, 2001; Rasmussen \& Lewandowski, 1998; Stewart \& Costerton, 2001). The phenomenon where bacteria are capable of unicellular existence, while residing within multicellular communities resembling the cells of higher organisms, has excited considerable research interest (HallStoodley \& Stoodley, 2002). Much of the information that we have today about biofilms has arisen from studies on Pseudomonas aeruginosa biofilms (Costerton et al., 1999; Gottenbos et al., 1999; Sauer et al., 2002). However, the ability to form biofilms is being identified in a number of other diverse organisms, of particular interest being pathogenic genera such as Vibrio, Escherichia, Salmonella, Listeria, Streptococcus, Staphylococcus, Klebsiella and Mycobacterium. The mycobacteria include pathogenic as well as non-pathogenic species (Rastogi et al., 2001). M. smegmatis, one of the non-pathogenic mycobacteria, was first isolated from smegma, a biofilm formed from the genital secretions of male mammals. It was discovered by Lustgarten in 1884 in syphilitic chancres and gummae (Lustgarten, 1884). Soon these bacteria were identified in the normal smegma by Alvarez and Tavel (Alvarez, 1885). It is now well established that M. smegmatis and some other non-tuberculous mycobacteria, such as Mycobacterium fortuitum and Mycobacterium marinum, can live and grow either planktonically or as a biofilm (Bardouniotis et al., 2003). In the laboratory scenario, Kolter and co-workers demonstrated that M. smegmatis is capable of forming biofilms on polyvinyl chloride surfaces, and that genes involved in synthesis and transport of glycopeptidolipids (GPLs) were essential for biofilm formation (Martinez et al., 1999; Recht et al., 2000; Recht \& Kolter, 2001). Ojha et al. (2005) recently showed that biofilm formation in $M$. smegmatis was associated with changes in the profile of mycolic acids - the characteristic long-chain fatty acids of mycobacterial cell wall. That article also demonstrated that the chaperone GroEL1 was a dedicated chaperone involved in this switch.

A characteristic feature of biofilm formation is that it is a dynamic process, and there is increasing evidence that it is influenced by complex regulatory pathways (Hall-Stoodley \& Stoodley, 2002). The shift from the planktonic to the biofilm mode of growth involves complex transitions in the gene expression pattern, which in turn are responsible for the different phenotypes that the adherent bacteria display, as has been identified in several bacterial systems including
M. smegmatis (Beloin \& Ghigo, 2005; O’Toole et al., 2000; Ojha et al., 2005). Even though the specific details of these networks are yet to be elucidated, the bacterial RNAP, which carries out the most basic as well as preliminary step of gene expression, can be expected to have a pivotal role in this process. There are several instances of the roles played by sigma factors in biofilm development in the literature, albeit with differing opinions. In $P$. aeruginosa biofilms, rpoS has been shown to be either repressed by two- to threefold or slightly activated (Whiteley et al., 2001; Xu et al., 2001). The stationary-phase sigma factor $r p o H$ is another upregulated gene in the same organism. However, reports on the role of Escherichia coli rpoS in biofilms remain much debated (Adams \& McLean, 1999; Corona-Izquierdo \& MembrilloHernandez, 2002; Schembri et al., 2003).

Here we report that the deletion mutation of $r p o Z$ encoding the $\omega$ subunit in M. smegmatis results in pleiotropic surface-associated phenotypes. In addition to the previously reported altered colony morphology these include compromised ability of the bacterium to form biofilm and reduction in the sliding motility characteristic of mycobacteria. Further characterization showed these phenotypes to be associated with a defect in establishing an extracellular matrix. Complementation of the mutant strain with an integrated copy of the wild-type $r p o Z$ gene restored the wild-type characteristics, thus implicating $\omega$ directly in the observed phenotypes. Chemical characterization of the mutant cell wall demonstrated a GPL profile identical to the wild-type, but a defect in the induction of short-chain mycolic acids, typical of transition to the biofilm mode of growth in M. smegmatis as recently reported.

\section{METHODS}

Bacterial strains and growth conditions. The parent wild-type strain of M. smegmatis used for construction of the knockout was $\mathrm{mc}^{2} 155$. Construction of strain mcdrz, with a deletion mutation of rpoZ in $\mathrm{mc}^{2} 155$, and strain mcdrzco (mcdrz complemented with a copy of the wild-type rpoZ gene integrated in the chromosome at the mycobacteriophage L5 integration site) has been described (Mathew et al., 2005). For planktonic cultures M. smegmatis was grown in Middlebrook 7H9 broth (Difco) supplemented with $0.05 \%$ Tween 80 and $2 \%$ glucose, or in Luria-Bertani (LB) broth. For examination of biofilm formation M. smegmatis was grown in Sauton's medium (containing, $\mathrm{g}^{-1}$ : ferric ammonium citrate $0 \cdot 0167$, L-aspargine $1 \cdot 33$, citric acid $0 \cdot 66$, magnesium sulphate $0 \cdot 166$, dipotassium hydrogen phosphate $0 \cdot 287$, sodium dihydrogen phosphate 0.633 , sodium chloride $0 \cdot 4$, Polysorbate- $800 \cdot 833$, and glycerol $2 \%$, w/v). Middlebrook $7 \mathrm{H} 9$ agar solid medium supplemented with $2 \%$ glucose was used as the solid medium for mycobacterial growth. Kanamycin $\left(30 \mu \mathrm{g} \mathrm{ml}^{-1}\right)$ and hygromycin $\left(50 \mu \mathrm{g} \mathrm{ml}^{-1}\right)$ were used as appropriate.

Biofilm formation assay. We adapted a previously described assay (O’Toole \& Kolter, 1998) to measure M. smegmatis biofilm formation. Initial observations of biofilms were carried out in $25 \mathrm{ml}$ polystyrene tissue culture flasks (Greiner). Five millilitres of Sauton's medium was added to the flasks and they were inoculated with 1:100 dilutions from a stationary-phase culture. The flasks were incubated in a humidified incubator and observed at intervals. 
For observing the surface pellicles, cultures were similarly inoculated and incubated in $30 \mathrm{ml}$ Sauton's medium in $90 \mathrm{~mm}$ diameter polystyrene Petri dishes.

For quantification of biofilm formation the method described by Friedman \& Kolter (2004) was adapted. Experiments were started from a fresh culture of bacteria grown to the early stationary phase in LB. This culture was then diluted to a final $\mathrm{OD}_{600}$ of 0.0025 in Sauton's medium and distributed into 96-well polystyrene plates at time zero. Each well received an inoculum of $200 \mu \mathrm{l}$. Eight wells were assayed for each strain at each time point, and the experiment was repeated three times. The samples were emptied from the wells, and the wells were washed with water by flushing them with a micropipette until all visible free-floating cells were removed. This process was carried out twice. The adherent film left behind was stained by the addition of $300 \mu 11 \%$ crystal violet solution and incubation for $20 \mathrm{~min}$. The wells were then again washed thoroughly and allowed to dry. The bound dye was quantified after incubating each well with $350 \mu \mathrm{l}$ DMSO for $1 \mathrm{~h}$ and subsequently measuring the $A_{590}$ using a microtitre plate reader. The $\mathrm{OD}_{600}$ of the untreated wells was also measured in the microtitre plate reader at different time points to assess the growth under these conditions.

Sliding motility. The technique for measuring the sliding motility of mycobacteria was adapted from Etienne et al. (2002). Briefly, Middlebrook $7 \mathrm{H} 9$ base medium (Difco) was solidified with $0 \cdot 3 \%$ high-grade agarose (Sigma). Plates were inoculated in their centre with $10 \mu \mathrm{l}$ of the cultures after adjusting the $\mathrm{OD}_{600}$ to $0 \cdot 5$. Spreading was evaluated after incubation for 5 days at $37^{\circ} \mathrm{C}$ in a humidified incubator.

Scanning electron microscopy (SEM). Biofilms grown for 7 days in tissue culture flasks as described above were fixed in a solution of $2 \cdot 5 \%$ glutaraldehyde in $0 \cdot 1 \mathrm{M}$ phosphate buffer, $\mathrm{pH} 7 \cdot 4$, overnight at $4{ }^{\circ} \mathrm{C}$. The samples were rinsed once in the same buffer and dehydrated by increasing concentrations of ethanol $(30 \%, 50 \%, 70 \%, 90 \%$ and $100 \%)$. This was followed by three changes of hexamethyldisilane and then the samples were dried in a fume hood. The dried samples were fixed to stubs with conductive self-adhesive carbon tapes, coated with gold film sputtering and used for analysis with an FEI Quanta-200 scanning electron microscope.

Purification and analysis of glycopeptidolipids (GPLs). The GPLs from the different strains of mycobacteria were purified and analysed by TLC and mass spectrometry as described previously (Mukherjee et al., 2005).

Hydrophobicity index. Relative hydrophobicities were assessed by the hexadecane partition procedure (Rosenberg et al., 1980).

Mycolic acid analysis. Biofilms were harvested from $\mathrm{mc}^{2} 155$ and mcdrz cultures grown for the required period of time in Petri dishes as mentioned above. The low amount of biofilms formed by mcdrz necessitated harvesting from 20 plates to obtain adequate starting material. The harvested films were refluxed overnight with $50 \mathrm{ml}$ tetrabutylammonium hydroxide $(15 \%)$ at $100{ }^{\circ} \mathrm{C}$, followed by addition of $50 \mathrm{ml}$ dichloromethane and $2.5 \mathrm{ml}$ methyl iodide and stirred at room temperature for $1 \mathrm{~h}$. After centrifugation at 10000 r.p.m. for $10 \mathrm{~min}$, the organic phase was separated and washed once with $0.2 \mathrm{M} \mathrm{HCl}$ and once with water, then dried under vacuum. The residue was resuspended in $5 \mathrm{ml}$ dichloromethane and spotted on a TLC plate, which was developed three times in petroleum ether/ ethyl acetate (95:5, v/v) (Kremer et al., 2000).

For mass spectrometric analysis, total mycolates were analysed with a MALDI-TOF-MS instrument (ULTRAFLEX TOF/TOF, Brucker Daltonics) equipped with a pulsed $\mathrm{N}_{2}$ laser. The sample was dissolved in chloroform/methanol $(2: 1, \mathrm{v} / \mathrm{v})$ and spotted as a $1: 1(\mathrm{v} / \mathrm{v})$ mixture with 2,5 dihydroxybenzoic acid as matrix and analysed in the reflectron mode using a $90 \mathrm{~ns}$ time delay, and a $25 \mathrm{kV}$ accelerating voltage in the positive ion mode. One thousand shots were averaged to obtain the spectrum.

\section{RESULTS}

\section{mcdrz is defective in biofilm formation}

It has been noted that bacterial colonies which grow on the surface of solid medium are examples of the biofilm mode of bacterial growth (Branda et al., 2005). The morphological changes shown by colonies reflect the variations in the extracellular matrix produced by the cells which constitute the colonies. With this in the background, we suspected that the strain mcdrz, which showed altered colony morphology, could show a difference in its biofilm-forming ability as well. On initial observation of biofilms using flask and Petri dish cultures (see Methods), we noted that mcdrz was defective in biofilm formation (Fig. 1). $\mathrm{mc}^{2} 155$ and mcdrzco cultures started forming pellicles at the air-liquid interface which were apparent as microcolonies floating on the surface after $24 \mathrm{~h}$ growth. These spread and coalesced to form a sturdy film covering the entire interface surface in 3-4 days after inoculation, followed by the film creeping up along the wall of the flask or dish (Fig. 1a, d, c, f). At 5-6 days after inoculation the characteristic wrinkled appearance of the surface of the films became distinctly apparent in the case of these two strains (Fig. 1g, i). The mcdrz culture lagged behind $\mathrm{mc}^{2} 155$ and mcdrzco cultures by about $24 \mathrm{~h}$ in forming apparent pellicles. Defective biofilm formation by mcdrz is evident from the appearance of the surface pellicles as well as the adherent growth (Fig. 1b, e, h). These defects were not corrected even after prolonged incubation. The amount of mcdrz film formed remained considerably less than the wild-type film and was either discontinuous or fragile enough to be broken up by slight disturbances, and completely lacked the surface wrinkles. Although the mutant film could climb up the wall of the flask, the degree to which it climbed was considerably less than the wild-type (Fig. 1b). The complemented strain mcdrzco formed biofilms which faithfully mimicked the wild-type films.

Strain mcdrz grows more slowly than the wild-type $\mathrm{mc}^{2} 155$ (Mathew et al., 2005). Hence it was possible that our result reflected a slowness of biofilm formation in the case of mcdrz, although even on prolonged incubation we did not observe mcdrz achieving a wild-type-like biofilm. Therefore we followed biofilm formation by all the three strains quantitatively for a longer period, to ascertain whether mcdrz would eventually achieve a similar biofilm density as the wild-type. Biofilm formation was followed for $240 \mathrm{~h}$. As can be seen from Fig. 2(a), the maximum amount of biofilm formed by mcdrz was less than the plateau achieved by $\mathrm{mc}^{2} 155$. Biofilm formation by mcdrzco followed that by $\mathrm{mc}^{2} 155$ closely. In addition, it can be seen that mcdrz biofilm formation has a slower slope than the wild-type or the complemented strains. $\mathrm{mc}^{2} 155$ 

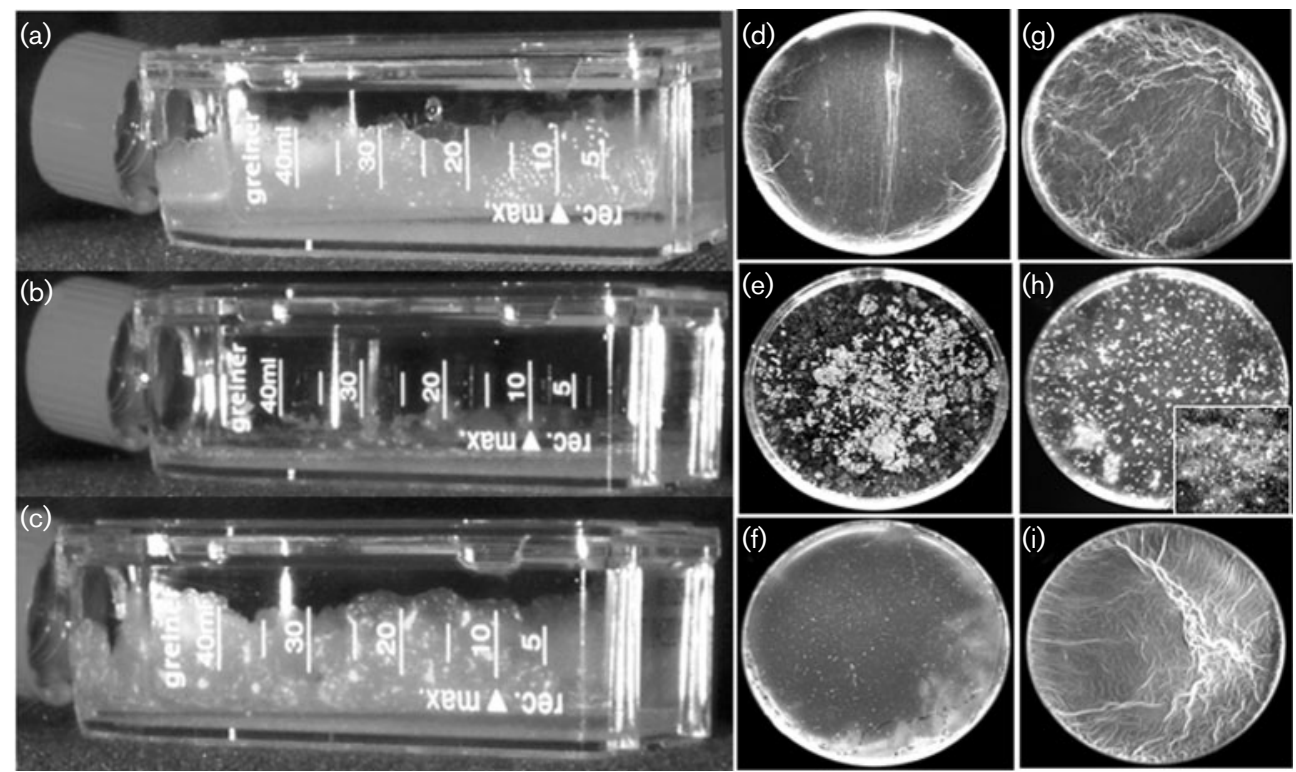

Fig. 1. Comparison of biofilm formation by $m c^{2} 155(a, d, g)$, mcdrz (b, e, h) and mcdrzco (c, $\left.f, i\right)$. The cultures were inoculated as described in Methods and the photographs were taken 4 days ( $d, e, f)$ and 7 days ( $, b, c, g, h, i)$ post-inoculation. The results shown are representative of observations made more than three times. The inset in $(h)$ is a close-up view of the same plate.

and mcdrzco reached a plateau of biofilm formation in 120-144 h whereas mcdrz formed a biofilm more slowly and reached a lower plateau in 144-168 h. The experiment was repeated three times with the same results. In addition, following the $\mathrm{OD}_{600}$ of the wells showed that the growth of mcdrz eventually attained densities comparable to that of $\mathrm{mc}^{2} 155$ in about $140-160 \mathrm{~h}$ time under these conditions. The data from a representative plate can be seen in Fig. 2(b).
Since the thorough washing step we employed in this assay effectively measured only the mature matrix-encased biofilm, we tried to investigate the initiation phase of biofilm formation following the method described for $P$. aeruginosa (Friedman \& Kolter, 2004). However, we were not able to obtain consistent values for the amount of cells adhering in the initiation phase using this method in the system we studied. Biofilms formed by mcdrz gave highly fluctuating values for film density in the initiation phase, possibly owing
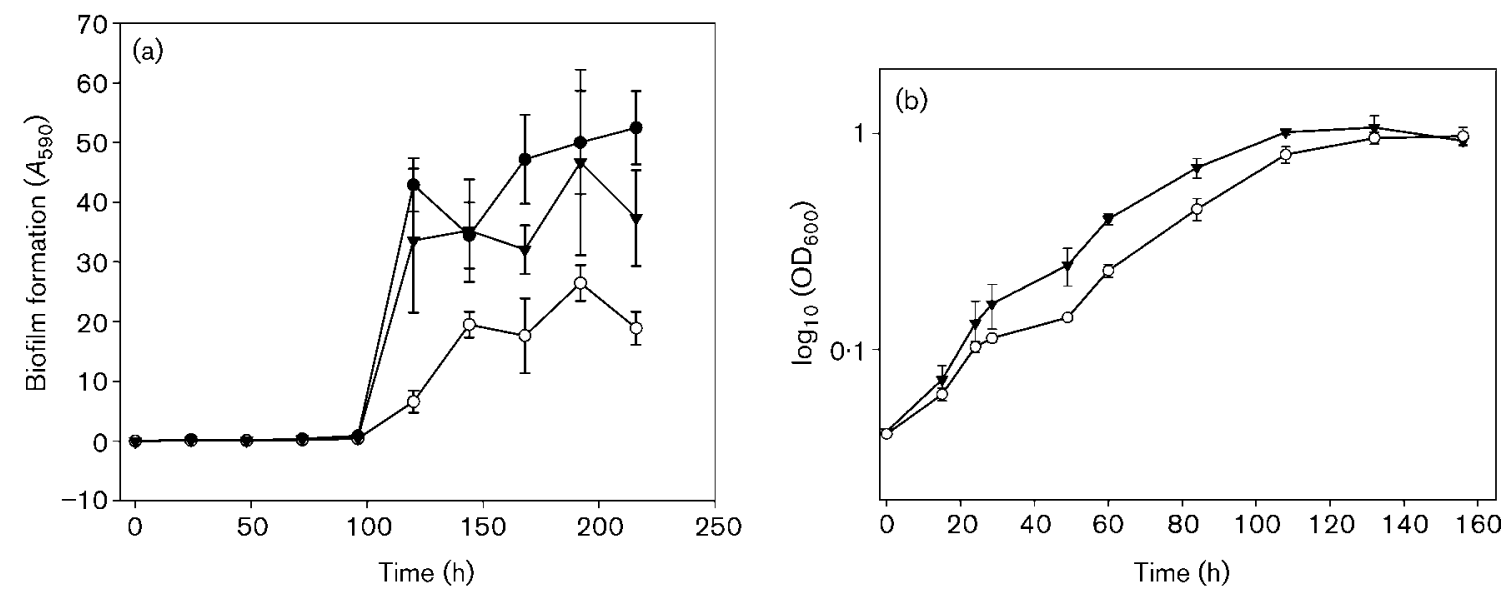

Fig. 2. (a) Biofilm formation of $\mathrm{mc}^{2} 155(\bullet)$, mcdrz $(\bigcirc)$ and mcdrzco $(\boldsymbol{\nabla})$ followed for $240 \mathrm{~h}$ by crystal violet staining assay. Each point represents the mean \pm SD of values from eight wells of a representative experiment. The experiment was repeated three times with the same results. (b) Comparison of $\mathrm{OD}_{600}$ values of $\mathrm{mc}^{2} 155(\boldsymbol{\nabla})$ and mcdrz $(\bigcirc)$ cultures grown in 96 -well plates. Each point represents the mean $\pm S D$ of values from eight wells of a representative experiment. 
to the fragile nature of the film generated by the mutant bacteria.

\section{mcdrz displays reduced sliding motility}

Although mycobacteria have been traditionally considered as non-motile (Goodfellow \& Cross, 1983), the ability of these bacteria to move by sliding over a solidified medium has been clearly demonstrated (Martinez et al., 1999). A relationship between inability to slide and a defect in biofilm formation has been shown in M. smegmatis mutants by Kolter's group (Recht et al., 2000). Looking for a converse relationship, we examined the ability of the biofilmdefective strain, mcdrz, to demonstrate sliding motility as described in Methods. The results were interesting, as we found that the mutant strain (Fig. 3b) had a reduced ability to display sliding motility compared to the wild-type (Fig. 3a), and as in the case of the biofilm defect, this inability was also rescued by complementation with a wild-type copy of rpoZ in mcdrzco (Fig. 3c). The smaller, denser zone in the centre of the plate represents surface colony growth, and it can be seen that the surrounding lighter hazy zone of motility is considerably reduced in the case of mcdrz (Fig. 3b).

\section{mcdrz has identical GPL profile and cell surface hydrophobicity to $\mathrm{mc}^{2} \mathbf{1 5 5}$}

While analysing the connection between sliding motility and biofilm formation, Kolter's group showed that both processes required the presence of GPLs (Recht et al., 2000). This, combined with many previous observations including those from our own laboratory that altered GPL profiles are associated with changes in colony morphology and motility in mycobacteria (Etienne et al., 2002; Ojha et al., 2002), prompted us to examine the GPL profile of mcdrz. TLC as well as mass spectrometric examination of the GPL populations from $\mathrm{mc}^{2} 155$ and mcdrz provided identical profiles (data not shown). Although we did not observe any difference in the GPL profile between mcdrz and the wild-type strain, we decided to compare the hydrophobic properties of the cell surfaces of the three strains. Hydrophobic interactions have been shown to be important in bacterial attachment to surfaces (Rosenberg \& Kjelleberg, 1986), and in mycobacteria, which do not have specialized appendages for attachment or motility, surface hydrophobicity could play a crucial role in adherence and biofilm formation as well as motility (Hall-Stoodley \& LappinScott, 1998; Recht et al., 2000). However, our experiments demonstrated similar hydrophobicity values for $\mathrm{mc}^{2} 155$ and mcdrz, thus excluding the possibility that the difference in phenotype we observed was due to differences in surface hydrophobocity (data not shown).

\section{SEM demonstrates that mcdrz is defective in extracellular matrix formation}

The results hitherto described give a picture of a reduced amount of biofilm adhering to the surface in the case of mcdrz, a deficiency that appears to be corrected in the complemented strain mcdrzco. Since cell surface hydrophobicity or GPL composition could not explain the observed phenotype, we examined the spatial distribution of bacteria within these films by SEM. The biofilms used for the experiment were grown for 7 days, by which time previous experiments have shown that biofilm formation by all the three stains reached a plateau. It is worth mentioning here that another difference in properties of the mcdrz biofilm with respect to the other two became apparent during the procedure of sample preparation for SEM. While $\mathrm{mc}^{2} 155$ and mcdrzco biofilms retained their structure as films until the end of the process, mcdrz biofilms broke down and crumbled during the dehydration steps and we were able to obtain only granular particles at the end of the process. This further demonstrated the fragility of mcdrz biofilms. Low-magnification pictures (Fig. 4) display this difference in appearance between $\mathrm{mc}^{2} 155$ and mcdrz

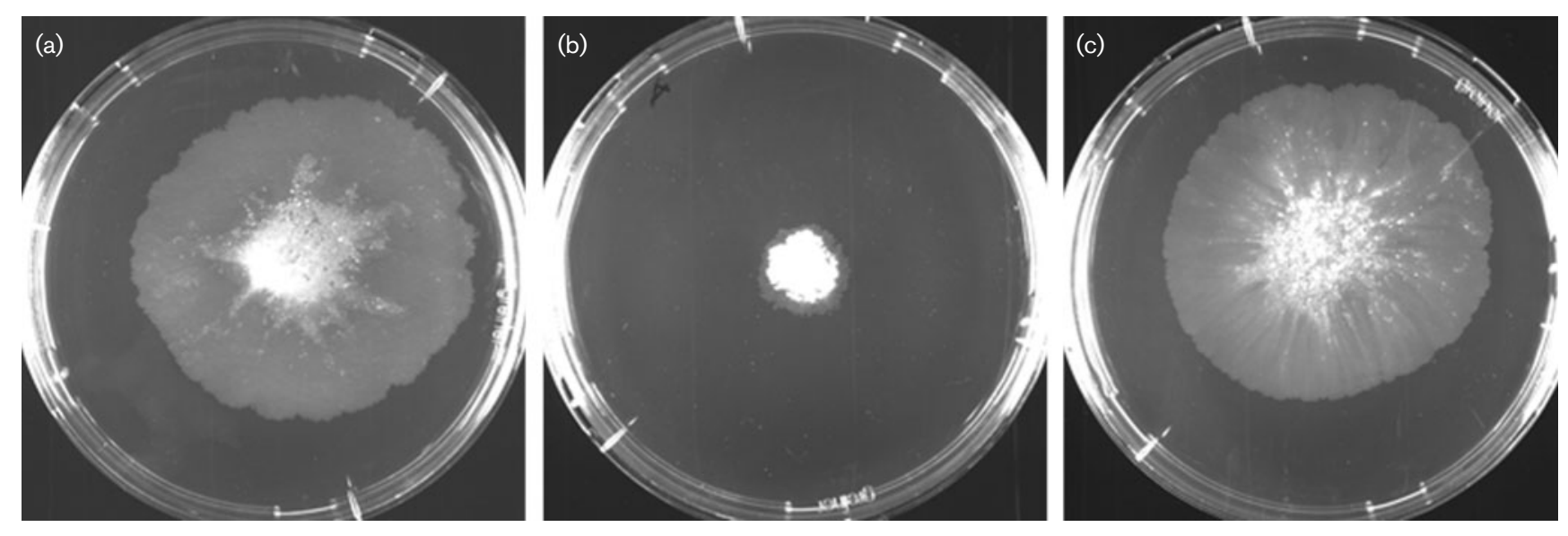

Fig. 3. Representative pictures of sliding motility of $\mathrm{mc}^{2} 155$ (a), mcdrz (b) and mcdrzco (c) on MB7H9-0.3\% agarose plates. The plates were photographed after 5 days of incubation. 

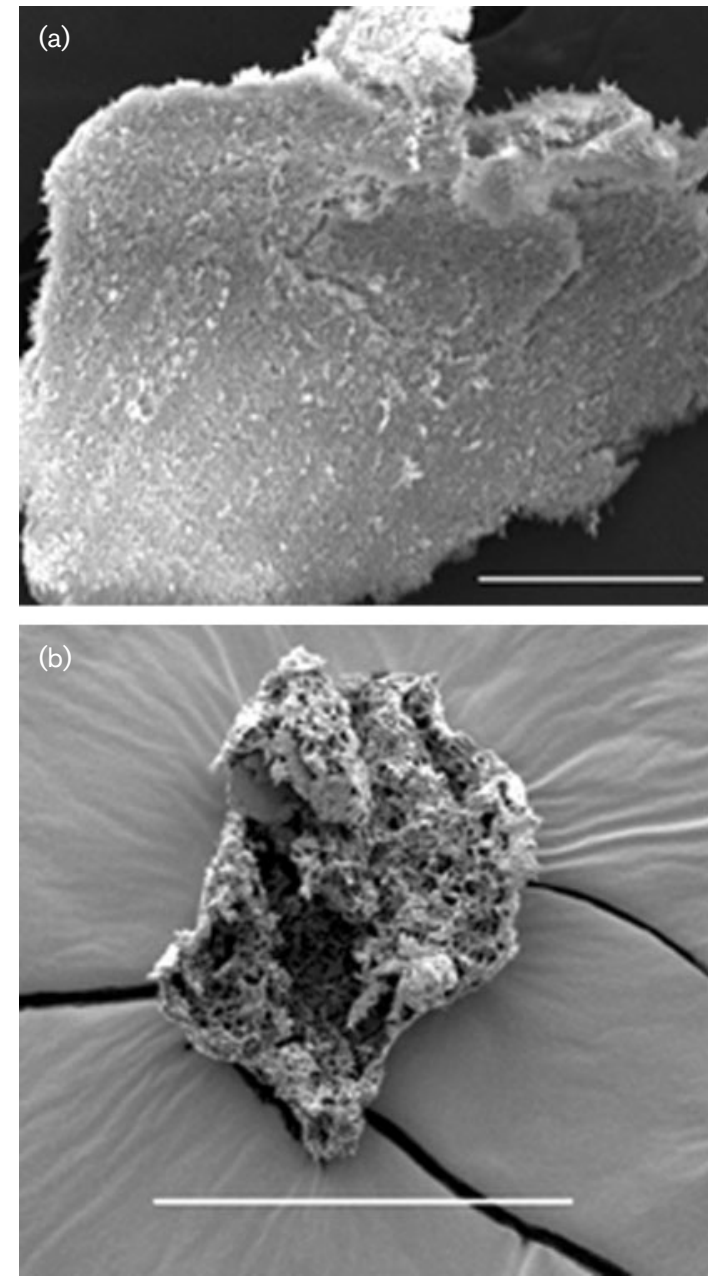

Fig. 4. Low-magnification SEM pictures of $\mathrm{mc}^{2} 155$ (a) and mcdrz (b). Fields shown are representative of independent biofilm preparations for SEM. Bars, $100 \mu \mathrm{m}$.

biofilms after processing for electron microscopy. A crumbled appearance and relatively loose arrangement of cells visible with numerous cavities distinguishes the modrz biofilm (Fig. 4b) from the smooth and tightly packed wildtype biofilm (Fig. 4a). The mcdrzco biofilm looked identical to the $\mathrm{mc}^{2} 155$ biofilm at this magnification.

The spatial arrangement of cells within the biofilm was evident at higher-magnification and resembled that in SEM pictures of other mycobacteria (Bardouniotis et al., 2003; Hall-Stoodley \& Lappin-Scott, 1998). Both $\mathrm{mc}^{2} 155$ (Fig. 5a) and mcdrzco (Fig. 5c) displayed thick mature biofilms with abundant extracellular matrix holding the rods together, interspersed with channels. The tendency of the bacilli to become arranged together into linear cord-like formations was apparent. In the case of mcdrz biofilms, although the cells were closely packed, the deficiency of extracellular matrix was clearly visible (Fig. 5b). There were only some patches of matrix holding cells together distributed over the
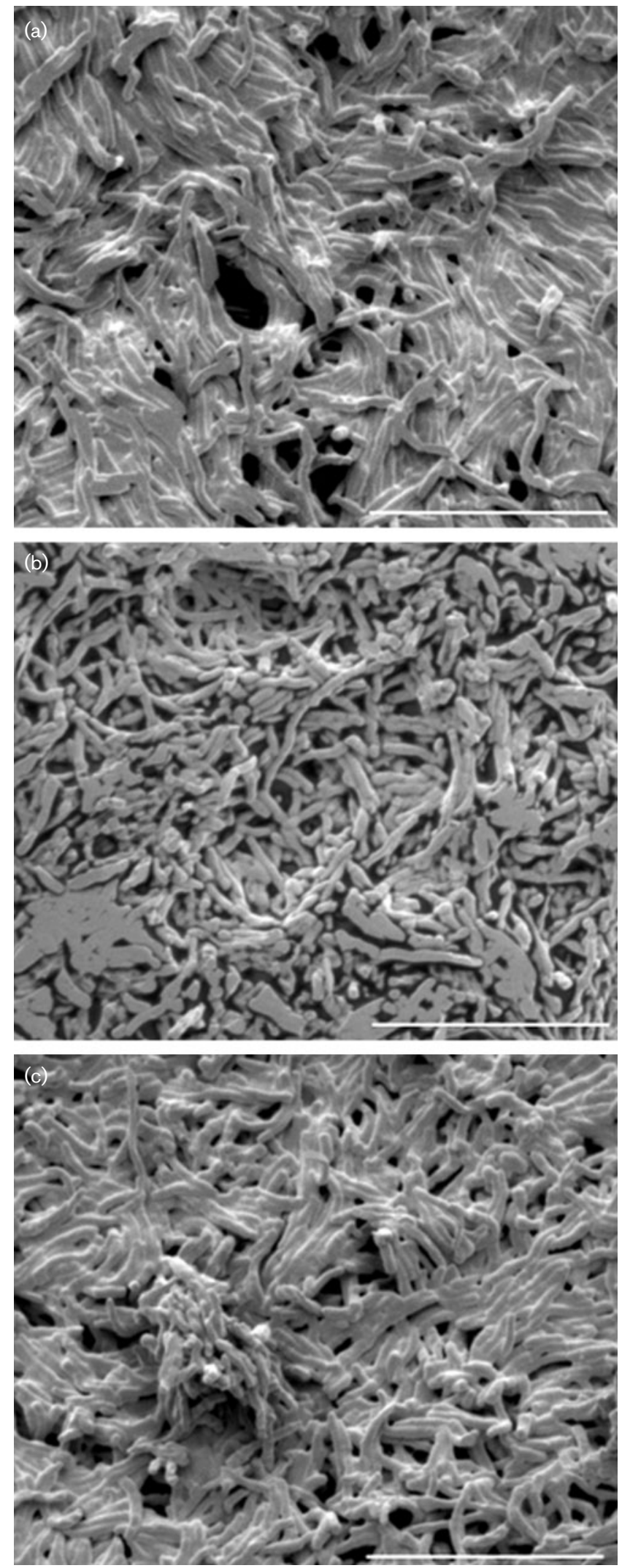

Fig. 5. High-magnification SEM pictures of $\mathrm{mc}^{2} 155$ (a), mcdrz (b) and mcdrzco (c). Fields shown are representative of independent biofilm preparations for SEM. Bars, $10 \mu \mathrm{m}$. 
film, and we have shown a field where a few such patches are visible.

\section{mcdrz shows a defect in transition to short-chain mycolates during biofilm growth}

Since the GPL constitution did not differ between the wildtype and rpoZ mutant, the composition of the other major chemical component of mycobacterial cell wall, namely mycolic acids, was investigated. Examination of mycolic acids from planktonic as well as biofilm cultures of both the bacteria by TLC failed to show any difference between the strains (data not shown). While this work was in progress, Ojha et al. (2005) reported characteristic changes in mycolic acid profile associated with biofilm development in M. smegmatis which were indiscernible during chromatography and became apparent only on mass spectrometric examination of the fatty acids. Upon mass spectrometric analysis we observed that at 4 days the wild-type profile displayed a predominance of short-chain mycolates as expected (Fig. 6a), while in the case of mcdrz, only longchain mycolates were seen after the same duration of biofilm growth (Fig. 6b). Since Ojha et al. (2005) carried out the examination after 4 days of biofilm growth, we followed the same time scale for our initial examination of mycolates. However, as we know that mcdrz cultures take about 7 days to form maximum amounts of biofilm, we grew the mutant biofilm for 7 days and carried out the same procedure. Even in this case, mcdrz biofilms showed only long-chain mycolic acids (Fig. 6c). Here it must be mentioned that we cannot conclude that the long-chain mycolic acids arise from the floating population of mcdrz alone; contamination from the bacteria settled below cannot be ruled out.

\section{DISCUSSION}

Here we show that deletion of the $\omega$ subunit of RNAP causes pleiotropic surface-related phenotypes in M. smegmatis. The deletion mutant mcdrz showed reduced sliding motility and defective biofilm formation. SEM demonstrated the failure to form biofilms to be associated with a deficiency in formation of extracellular matrix by the mcdrz bacterial population. Complementation of the mutant strain with a copy of the wild-type rpoZ gene integrated in the bacterial chromosome restored the wildtype phenotypes, proving that the lack of $\omega$ subunit was indeed responsible for the phenotypic changes observed in mcdrz.

In M. smegmatis, mutants in the GPL biosynthetic pathway have been demonstrated to have deficiencies in biofilm

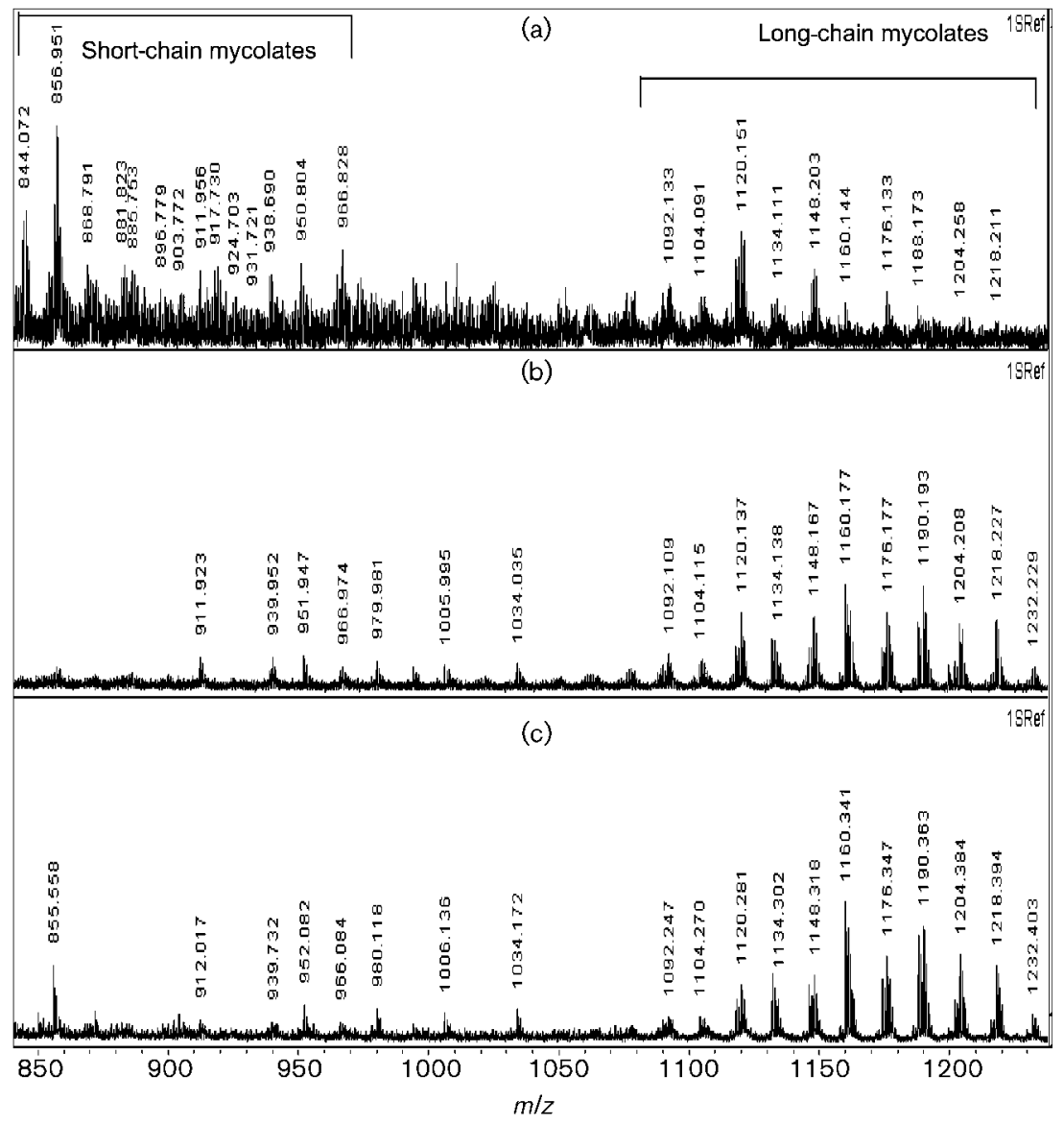

Fig. 6. Mass spectrometric comparison of total mycolates extracted from 4-day-old $\mathrm{mc}^{2} 155$ biofilm (a), 4-day-old mcdrz biofilm (b) and 7-day-old mcdrz biofilm (c). 
formation as well as sliding motility. But we observed similar GPL profiles for $\mathrm{mc}^{2} 155$ and mcdrz. However, even as they elucidated sliding motility and its connection to GPLs in $M$. smegmatis and Mycobacterium avium, Kolter and coworkers pointed out the possibility that GPLs were not likely to be the only components affecting mycobacterial spreading motility (Martinez et al., 1999). They observed a mucoid clear material or slime layer of unknown composition surrounding the spreading bacteria, and also differences in spreading phenotypes of M. avium 2151-SmD and 2151-SmT, which were identical in their GPL profile (Belisle et al., 1993), but differed in the amount of capsular polysaccharide (Rastogi et al., 1981). The deficiency in extracellular matrix can probably explain both the reduced sliding motility and the defective biofilm formation which were observed in strain mcdrz; it seems likely that the components of the extracellular matrix also facilitate bacterial sliding on solid surfaces.

In the case of biofilm formation, extracellular matrix has a pre-eminent role, with its presence being demonstrated in all the biofilms studied so far. The chemical components of the extracellular matrix can be polysaccharides, nucleic acids and proteins, extracellular DNA and possibly even the debris left behind by the dead cells (Flemming et al., 2000; Sutherland, 2001; Webb et al., 2003; Whitchurch et al., 2002; Yarwood et al., 2004). However, our knowledge about the diverse components of extracellular matrix is still very limited, with almost nothing being known about the extracellular matrix of mycobacteria. In contrast to most bacteria which produce robust biofilms, mycobacteria do not produce exopolysaccharides. Given the important role of short-chain mycolic acids in developing the architecture of $M$. smegmatis biofilms and our observation that the lack of these mycolic acids coincides with defective extracellular matrix formation, it seems reasonable to suspect that mycolic acids may contribute to the process of matrix formation.

An explanation for the pleiotropic effects arising from $r p o Z$ deletion is not apparent immediately. It has been noted in our laboratory that in an E. coli rpoZ null mutant, large amounts of GroEL are recruited by RNAP (Mukherjee et al., 1999). Since one of the chaperone proteins in $M$. smegmatis, GroEL1, has been shown to be a dedicated chaperone involved in synthesis of mycolic acids during biofilm formation, we are interested in analysing the additional proteins which we have observed to co-purify with RNAP when the enzyme is purified from mcdrz.

The conditions faced by bacteria residing inside biofilms have been shown to be considerably different from those existing during planktonic growth (Huang et al., 1998; Prigent-Combaret et al., 1999, 2001). The interior of a biofilm presents a stressful environment for the cells to grow owing to the microaerobic conditions, reduced availability of nutrients, and fluctuations in $\mathrm{pH}$ and osmotic pressure. In E. coli, a significant part of the biofilm response involves stationary-phase-induced genes (Beloin et al.,
2004; Schembri et al., 2003). In Bacillus subtilis, a large number of the genes induced during sporulation, a phenomenon provoked by starvation conditions, are activated during biofilm formation also (Ren et al., 2004). Upregulation of stress response regulators has also been noted in Streptococcus mutans and Staphylococcus aureus biofilm formation (Adams \& McLean, 1999; Knobloch et al., 2001; Rachid et al., 2000; Yoshida \& Kuramitsu, 2002), although this is yet another disputed topic (Corona-Izquierdo \& Membrillo-Hernandez, 2002; Kies et al., 2001; Valle et al., 2003). These findings, combined with the observations about the connections between stationary-phase sigma factors and biofilm development, make a strong case for the similarities that biofilm growth conditions have with conditions that prevail in stationary-phase (planktonic) cultures. In E. coli $\omega$ has been shown to be upregulated in the stationary phase of growth (Jishage \& Ishihama, 1998), and hence can be suspected to have a role in assisting the polymerase in transcribing the DNA under the stressful stationary-phase conditions. We would like to hypothesize that in the absence of $\omega$, the RNAP, alone or in association with any compensatory mechanism the cell has induced, undergoes structural alterations affecting the binding of one or a set of sigma factors which are not essential for survival, but have a role in development of biofilm in M. smegmatis. Indeed, it has been shown in the case of E. coli that RNAP isolated from an $r p o Z$ deleted strain shows altered sigma-binding properties (Mukherjee \& Chatterji, 1999).

An alternative and attractive possibility arises from the recent confirmation of the role of the $\omega$ subunit in mediating the effect of the stringent response on RNAP (Vrentas et al., 2005). The role of the stringent response in the development of biofilms has been shown for many organisms, including Listeria monocytogenes (Taylor et al., 2002), E. coli (Balzer \& McLean, 2002) and Streptococcus mutans (Lemos et al., 2004). This connection is not surprising, as adaptation to environmental changes and reduced availability of nutrients through the stringent response can be expected to play an important role in the establishment of a biofilm community. Given the role identified for $\omega$ in mediation of the effects of alarmone ppGpp on RNAP, the defective execution of stringent response signals for biofilm formation due to lack of $\omega$ subunit in RNAP can be conceived as a possible explanation for the phenotypes we observed for mcdrz. This will be similar to the explanation that has been offered for another surprising connection observed for the $\omega$ subunit: with antibiotic production and morphogenesis in Streptomyces kasugaensis (Kojima et al., 2002).

To conclude, our observations add to the limited literature available on the properties of M. smegmatis biofilm and also contribute to the known complexities of biofilm development and its regulation in general. In addition they suggest the possibility of physiological roles for the $\omega$ subunit which are still not elucidated. 


\section{ACKNOWLEDGEMENTS}

The authors wish to thank the Department of Biotechnology, Government of India, for financial support, and Keshab Darai, for technical help in SEM studies. Raju Mukherjee was supported by the SRF from CSIR, India.

\section{REFERENCES}

Adams, J. L. \& McLean, R. J. (1999). Impact of rpoS deletion on Escherichia coli biofilms. Appl Environ Microbiol 65, 4285-4287.

Alvarez, E. T. (1885). Recherches sur le bacille de Lustgarten. Arch Physiol Normal Pathol303-321.

Balzer, G. J. \& McLean, R. J. (2002). The stringent response genes relA and spoT are important for Escherichia coli biofilms under slow-growth conditions. Can J Microbiol 48, 675-680.

Bardouniotis, E., Ceri, H. \& Olson, M. E. (2003). Biofilm formation and biocide susceptibility testing of Mycobacterium fortuitum and Mycobacterium marinum. Curr Microbiol 46, 28-32.

Belisle, J. T., McNeil, M. R., Chatterjee, D., Inamine, J. M. \& Brennan, P. J. (1993). Expression of the core lipopeptide of the glycopeptidolipid surface antigens in rough mutants of Mycobacterium avium. J Biol Chem 268, 10510-10516.

Beloin, C. \& Ghigo, J. M. (2005). Finding gene-expression patterns in bacterial biofilms. Trends Microbiol 13, 16-19.

Beloin, C., Valle, J., Latour-Lambert, P. \& 8 other authors (2004). Global impact of mature biofilm lifestyle on Escherichia coli K-12 gene expression. Mol Microbiol 51, 659-674.

Branda, S. S., Gonzalez-Pastor, J. E., Ben-Yehuda, S., Losick, R. \& Kolter, R. (2001). Fruiting body formation by Bacillus subtilis. Proc Natl Acad Sci U S A 98, 11621-11626.

Branda, S. S., Vik, S., Friedman, L. \& Kolter, R. (2005). Biofilms: the matrix revisited. Trends Microbiol 13, 20-26.

Burgess, R. R. (1969). Separation and characterization of the subunits of ribonucleic acid polymerase. J Biol Chem 244, 6168-6176.

Christensen, B. B., Sternberg, C., Andersen, J. B., Palmer, R. J., Jr, Nielsen, A. T., Givskov, M. \& Molin, S. (1999). Molecular tools for study of biofilm physiology. Methods Enzymol 310, 20-42.

Corona-Izquierdo, F. P. \& Membrillo-Hernandez, J. (2002). A mutation in rpoS enhances biofilm formation in Escherichia coli during exponential phase of growth. FEMS Microbiol Lett 211, 105-110.

Costerton, J. W., Stewart, P. S. \& Greenberg, E. P. (1999). Bacterial biofilms: a common cause of persistent infections. Science 284, 1318-1322.

Davey, M. E. \& O'Toole, G. A. (2000). Microbial biofilms: from ecology to molecular genetics. Microbiol Mol Biol Rev 64, 847-867.

Etienne, G., Villeneuve, C., Billman-Jacobe, H., Astarie-Dequeker, C., Dupont, M. A. \& Daffé, M. (2002). The impact of the absence of glycopeptidolipids on the ultrastructure, cell surface and cell wall properties, and phagocytosis of Mycobacterium smegmatis. Microbiology 148, 3089-3100.

Flemming, H. C. W. J., Mayer, C., Korstgens, V. \& Borchard, W. (2000). Cohesiveness in biofilm matrix polymers. In Community Structure and Co-operation in Biofilms (SGM Symposium Series vol. 59), pp. 87-105 Cambridge: Cambridge University Press.

Friedman, L. \& Kolter, R. (2004). Genes involved in matrix formation in Pseudomonas aeruginosa PA14 biofilms. Mol Microbiol 51, 675-690.

Ghosh, P., Ishihama, A. \& Chatterii, D. (2001). Escherichia coli RNA polymerase subunit omega and its $\mathrm{N}$-terminal domain bind fulllength $\beta$ ' to facilitate incorporation into the $\alpha 2 \beta$ subassembly. Eur J Biochem 268, 4621-4627.
Goodfellow, M. \& Cross, T. (1983). Classification. London: Academic Press.

Gottenbos, B., van der Mei, H. C. \& Busscher, H. J. (1999). Models for studying initial adhesion and surface growth in biofilm formation on surfaces. Methods Enzymol 310, 523-534.

Hall-Stoodley, L. \& Lappin-Scott, H. (1998). Biofilm formation by the rapidly growing mycobacterial species Mycobacterium fortuitum. FEMS Microbiol Lett 168, 77-84.

Hall-Stoodley, L. \& Stoodley, P. (2002). Developmental regulation of microbial biofilms. Curr Opin Biotechnol 13, 228-233.

Huang, C. T., Xu, K. D., McFeters, G. A. \& Stewart, P. S. (1998). Spatial patterns of alkaline phosphatase expression within bacterial colonies and biofilms in response to phosphate starvation. Appl Environ Microbiol 64, 1526-1531.

Jishage, M. \& Ishihama, A. (1998). A stationary phase protein in Escherichia coli with binding activity to the major sigma subunit of RNA polymerase. Proc Natl Acad Sci U S A 95, 4953-4958.

Kies, S., Otto, M., Vuong, C. \& Gotz, F. (2001). Identification of the sigB operon in Staphylococcus epidermidis: construction and characterization of a sigB deletion mutant. Infect Immun 69, 7933-7936.

Knobloch, J. K., Bartscht, K., Sabottke, A., Rohde, H., Feucht, H. H. \& Mack, D. (2001). Biofilm formation by Staphylococcus epidermidis depends on functional RsbU, an activator of the sigB operon: differential activation mechanisms due to ethanol and salt stress. J Bacteriol 183, 2624-2633.

Kojima, I., Kasuga, K., Kobayashi, M., Fukasawa, A., Mizuno, S., Arisawa, A. \& Akagawa, H. (2002). The rpoZ gene, encoding the RNA polymerase omega subunit, is required for antibiotic production and morphological differentiation in Streptomyces kasugaensis. J Bacteriol 184, 6417-6423.

Kremer, L., Douglas, J. D., Baulard, A. R. \& 9 other authors (2000). Thiolactomycin and related analogues as novel anti-mycobacterial agents targeting KasA and KasB condensing enzymes in Mycobacterium tuberculosis. J Biol Chem 275, 16857-16864.

Lemos, J. A., Brown, T. A., Jr \& Burne, R. A. (2004). Effects of RelA on key virulence properties of planktonic and biofilm populations of Streptococcus mutans. Infect Immun 72, 1431-1440.

Lustgarten, S. (1884). Ueber spezifische Bacillen in syphilitischen Krankheitsprodukten. Wiener Medizinische Wochenschrift 1.

Mah, T. F. \& O'Toole, G. A. (2001). Mechanisms of biofilm resistance to antimicrobial agents. Trends Microbiol 9, 34-39.

Martinez, A., Torello, S. \& Kolter, R. (1999). Sliding motility in mycobacteria. J Bacteriol 181, 7331-7338.

Mathew, R., Ramakanth, M. \& Chatterji, D. (2005). Deletion of the gene rpoZ, encoding the omega subunit of RNA polymerase, in Mycobacterium smegmatis results in fragmentation of the $\beta$ ' subunit in the enzyme assembly. J Bacteriol 187, 6565-6570.

Minakhin, L., Bhagat, S., Brunning, A., Campbell, E. A., Darst, S. A., Ebright, R. H. \& Severinov, K. (2001). Bacterial RNA polymerase subunit omega and eukaryotic RNA polymerase subunit RPB6 are sequence, structural, and functional homologs and promote RNA polymerase assembly. Proc Natl Acad Sci U S A 98, 892-897.

Mukherjee, K. \& Chatterji, D. (1999). Alteration in template recognition by E. coli RNA polymerase lacking the omega subunit: a mechanistic analysis through gel retardation and foot-printing studies. J Biosci 24, 453-459.

Mukherjee, K., Nagai, H., Shimamoto, N. \& Chatterji, D. (1999). GroEL is involved in activation of Escherichia coli RNA polymerase devoid of the omega subunit in vivo. Eur J Biochem 266, 228-235.

Mukherjee, R., Gomez, M., Jayaraman, N., Smith, I. \& Chatterji, D. (2005). Hyperglycosylation of glycopeptidolipid of Mycobacterium 
smegmatis under nutrient starvation: structural studies. Microbiology 151, 2385-2392.

Ojha, A. K., Varma, S. \& Chatterji, D. (2002). Synthesis of an unusual polar glycopeptidolipid in glucose-limited culture of Mycobacterium smegmatis. Microbiology 148, 3039-3048.

Ojha, A., Anand, M., Bhatt, A., Kremer, L., Jacobs, W. R., Jr \& Hatfull, G. F. (2005). GroEL1: a dedicated chaperone involved in mycolic acid biosynthesis during biofilm formation in mycobacteria. Cell 123, 861-873.

O'Toole, G. A. \& Kolter, R. (1998). Initiation of biofilm formation in Pseudomonas fluorescens WCS365 proceeds via multiple, convergent signalling pathways: a genetic analysis. Mol Microbiol 28, 449-461.

O'Toole, G., Kaplan, H. B. \& Kolter, R. (2000). Biofilm formation as microbial development. Annu Rev Microbiol 54, 49-79.

Potera, C. (1996). Biofilms invade microbiology. Science 273, 1795-1797.

Prigent-Combaret, C., Vidal, O., Dorel, C. \& Lejeune, P. (1999). Abiotic surface sensing and biofilm-dependent regulation of gene expression in Escherichia coli. J Bacteriol 181, 5993-6002.

Prigent-Combaret, C., Brombacher, E., Vidal, O., Ambert, A., Lejeune, P., Landini, P. \& Dorel, C. (2001). Complex regulatory network controls initial adhesion and biofilm formation in Escherichia coli via regulation of the $\operatorname{csg} D$ gene. J Bacteriol 183, 7213-7223.

Rachid, S., Ohlsen, K., Wallner, U., Hacker, J., Hecker, M. \& Ziebuhr, W. (2000). Alternative transcription factor $\sigma^{\mathrm{B}}$ is involved in regulation of biofilm expression in a Staphylococcus aureus mucosal isolate. J Bacteriol 182, 6824-6826.

Rasmussen, K. \& Lewandowski, Z. (1998). Microelectrode measurements of local mass transport rates in heterogeneous biofilms. Biotechnol Bioeng 59, 302-309.

Rastogi, N., Frehel, C., Ryter, A., Ohayon, H., Lesourd, M. \& David, H. L. (1981). Multiple drug resistance in Mycobacterium avium: is the wall architecture responsible for exclusion of antimicrobial agents? Antimicrob Agents Chemother 20, 666-677.

Rastogi, N., Legrand, E. \& Sola, C. (2001). The mycobacteria: an introduction to nomenclature and pathogenesis. Rev Sci Tech 20, 21-54.

Recht, J. \& Kolter, R. (2001). Glycopeptidolipid acetylation affects sliding motility and biofilm formation in Mycobacterium smegmatis. $J$ Bacteriol 183, 5718-5724.

Recht, J., Martinez, A., Torello, S. \& Kolter, R. (2000). Genetic analysis of sliding motility in Mycobacterium smegmatis. J Bacteriol 182, 4348-4351.

Ren, D., Bedzyk, L. A., Setlow, P., Thomas, S. M., Ye, R. W. \& Wood, T. K. (2004). Gene expression in Bacillus subtilis surface biofilms with and without sporulation and the importance of $y v e R$ for biofilm maintenance. Biotechnol Bioeng 86, 344-364.

Rosenberg, M. \& Kjelleberg, S. (1986). Hydrophobic interactions in bacterial adhesion. Adv Microb Ecol 9, 353-393.
Rosenberg, M., Gutnick, D. \& Rosenberg, E. (1980). Adherence of bacteria to hydrocarbons: a simple method for measuring cell-surface hydrophobicity. FEMS Microbiol Lett 9, 29-33.

Sauer, K., Camper, A. K., Ehrlich, G. D., Costerton, J. W. \& Davies, D. G. (2002). Pseudomonas aeruginosa displays multiple phenotypes during development as a biofilm. J Bacteriol 184, 1140-1154.

Schembri, M. A., Kjaergaard, K. \& Klemm, P. (2003). Global gene expression in Escherichia coli biofilms. Mol Microbiol 48, 253-267.

Stewart, P. S. \& Costerton, J. W. (2001). Antibiotic resistance of bacteria in biofilms. Lancet 358, 135-138.

Sutherland, I. W. (2001). The biofilm matrix - an immobilized but dynamic microbial environment. Trends Microbiol 9, 222-227.

Taylor, C. M., Beresford, M., Epton, H. A., Sigee, D. C., Shama, G., Andrew, P. W. \& Roberts, I. S. (2002). Listeria monocytogenes relA and $h p t$ mutants are impaired in surface-attached growth and virulence. J Bacteriol 184, 621-628.

Valle, J., Toledo-Arana, A., Berasain, C., Ghigo, J. M., Amorena, B., Penades, J. R. \& Lasa, I. (2003). SarA and not $\sigma^{\mathrm{B}}$ is essential for biofilm development by Staphylococcus aureus. Mol Microbiol 48, 1075-1087.

Vrentas, C. E., Gaal, T., Ross, W., Ebright, R. H. \& Gourse, R. L. (2005). Response of RNA polymerase to ppGpp: requirement for the $\omega$ subunit and relief of this requirement by DksA. Genes Dev 19, 2378-2387.

Webb, J. S., Thompson, L. S., James, S., Charlton, T., TolkerNielsen, T., Koch, B., Givskov, M. \& Kjelleberg, S. (2003). Cell death in Pseudomonas aeruginosa biofilm development. J Bacteriol 185, 4585-4592.

Whitchurch, C. B., Tolker-Nielsen, T., Ragas, P. C. \& Mattick, J. S. (2002). Extracellular DNA required for bacterial biofilm formation. Science 295, 1487.

Whiteley, M., Bangera, M. G., Bumgarner, R. E., Parsek, M. R., Teitzel, G. M., Lory, S. \& Greenberg, E. P. (2001). Gene expression in Pseudomonas aeruginosa biofilms. Nature 413, 860-864.

Xu, K. D., Franklin, M. J., Park, C. H., McFeters, G. A. \& Stewart, P. S. (2001). Gene expression and protein levels of the stationary phase sigma factor, RpoS, in continuously-fed Pseudomonas aeruginosa biofilms. FEMS Microbiol Lett 199, 67-71.

Yarwood, J. M., Bartels, D. J., Volper, E. M. \& Greenberg, E. P. (2004). Quorum sensing in Staphylococcus aureus biofilms. J Bacteriol 186, 1838-1850.

Yildiz, F. H. \& Schoolnik, G. K. (1999). Vibrio cholerae O1 El Tor: identification of a gene cluster required for the rugose colony type, exopolysaccharide production, chlorine resistance, and biofilm formation. Proc Natl Acad Sci U S A 96, 4028-4033.

Yoshida, A. \& Kuramitsu, H. K. (2002). Multiple Streptococcus mutans genes are involved in biofilm formation. Appl Environ Microbiol 68, 6283-6291.

Zhang, G., Campbell, E. A., Minakhin, L., Richter, C., Severinov, K. \& Darst, S. A. (1999). Crystal structure of Thermus aquaticus core RNA polymerase at $3 \cdot 3 \AA$ resolution. Cell $\mathbf{9 8}, 811-824$. 